\title{
Efecto de la aplicación de un protocolo para pie diabético en un servicio de cirugía general
}

\author{
Rafael Sellés-Dechent ${ }^{1}$, Zutoia Balciscueta-Coltell ${ }^{1}$, \\ Inmaculada Torrijo-Gómez y Carmen Muñoz-Alonso ${ }^{2}$
}

'Servicio de Cirugía Genera Hospital Arnau de Vilanova. ${ }^{2}$ Servicio de Cirugía general Hospital de Llíria. Valencia España.

Recibido 2020-05-29y aceptado 2020-08-19

Correspondencia a: Dr. Rafael Sellés-Dechent rafaselles@yahoo.es

\section{Effect of the application of a diabetic protocol in a general surgery service}

Aim: The main objective in the patient with diabetic foot is to avoid amputation, thus, this study pretended to analyze the effect produced by the introduction of a protocol for the diabetic foot in our Department of Health. Materials and Method: It is a retrospective study. Age, sex, amputation and re-amputations rate in the first month, the average stay and the hospital re-entry were evaluated, the request for specific complementary analytical and radiological tests (blood test analytics with glycosylated hemoglobin and CT/MR/doppler arterial limb angiography) and patients remitted to the reference center to assess revascularization. All these data were analyzed comparing the patients admitted with diagnosis of diabetic foot in the period before to the implementation of the protocol (years 2010 to 2013) with those of the subsequent period (years 2014 to 2018). Results: Homogeneous groups were compared by age and sex. The result of the analysis was a significant reduction in the rate of amputations (172 versus 111, average of $22.2 \pm 9.9$ ), re-amputations (41 versus 15) and re-entry due to poor evolution in the first month (18 versus 7 ). No changes were found in the number of complementary radiological tests performed and the number of patients remitted to the reference hospital to assess revascularization. Conclusions: In the multidisciplinary management, the general surgeon is one of the important pillars. The implementation of an action protocol for the diabetic foot significantly reduces the amputation rate.

Key words: diabetic foot; protocol; amputation.

\section{Resumen}

Objetivo: El fin principal en el paciente con pie diabético es evitar la amputación, por ello, este estudio pretendió analizar el efecto que produjo la instauración de un protocolo de actuación para el pie diabético en nuestro departamento de salud. Materiales y Método: Se trata de un estudio retrospectivo. Se evaluó la edad, el sexo, la tasa de amputaciones y re-amputaciones en el primer mes, la estancia media y la añadida por reingreso para reamputación, la solicitud de pruebas analíticas y radiológicas complementarias específicas (analítica de sangre completa con hemoglobina glicosilada y angioTC/ $\mathrm{RM}$ /doppler arterial de miembros inferiores), y pacientes derivados al centro de referencia para valorar revascularización. Todos estos datos se analizaron comparando los pacientes ingresados con diagnóstico de pie diabético en el periodo previo a la implantación del protocolo (años 2010 a 2013), con los del periodo posterior (años 2014 a 2018). Resultados: Se compararon grupos homogéneos en cuanto a edad y sexo. El resultado del análisis fue una reducción estadísticamente significativa de la tasa de amputaciones (172 frente a 111 , media de $22,2 \pm 9,9)$, reamputaciones (41 frente a 15 ) y de reingreso (18 frente a 7) por mala evolución en el primer mes. No se encontraron cambios en el número de pruebas complementarias radiológicas realizadas y el número de pacientes remitidos a hospital de referencia para valorar revascularización. Conclusiones: Dentro del manejo multidisciplinar, el cirujano general es uno de los pilares importantes. La implantación de un protocolo de actuación para el pie diabético reduce de forma significativa la tasa de amputaciones.

Palabras clave: pie diabético; protocolo; amputación. 


\section{Introducción}

Se considera que un $15 \%$ de pacientes diabéticos, en algún momento de su vida, presentarán una úlcera y de estos, un $25 \%$ precisará algún tipo de amputación, por este motivo, la finalidad principal en la atención de los pies de los pacientes afectados de diabetes mellitus (DM) es evitar una amputación'1. De acuerdo con el estudio Eurodiale, el 58\% de las personas con DM que se presentan con una nueva úlcera del pie, tienen evidencia clínica de infección. Además, se estima que la infección del pie es la causa más frecuente de hospitalización relacionada con la DM y representa un factor importante para la amputación de la extremidad inferior ${ }^{2,3}$. El objetivo principal de nuestro estudio fue analizar el efecto que produjo la instauración de un protocolo de actuación para el pie diabético (PD) en nuestro departamento de salud.

\section{Materiales y Método}

Se trata de un estudio retrospectivo que analizó las siguientes variables: la edad, el sexo, la tasa de amputaciones y re-amputaciones en el primer mes, la estancia media, la añadida por reingreso para reamputación, la solicitud de pruebas complementarias específicas (analítica sanguínea con $\mathrm{HbAlc}$ $\mathrm{y}$ angioTC/RM/doppler arterial de miembros inferiores) y pacientes derivados al centro de referencia para valorar revascularización, todo ello comparando los pacientes ingresados con diagnóstico de PD en el periodo previo a la implantación del protocolo (años 2010 a 2013) con el periodo posterior (años 2014 a 2018).

Se realiza un protocolo de actuación para el PD que consta de 4 fases: 1) manejo en urgencias; 2) manejo en ingreso; 3) derivación del paciente susceptible de revascularización y, por último, 4) selección del nivel de amputación en el paciente no susceptible de revascularización. Es un protocolo dinámico en el que, con el tiempo, se ha ido añadiendo o modificando aspectos tras su instauración.

\section{Fase 1: manejo en urgencias}

- Anamnesis y antecedentes médico-quirúrgicos.

- Exploración física.

*Circulación: palpación de pulsos arteriales (femoral, poplíteo, tibial posterior y pedio).

*Sensibilidad: test de sensibilidad con monofilamento para descartar neuropatía.
*Infección: explorar la piel, las partes blandas y realizar el test probe to bone para descartar osteomielitis.

- Pruebas básicas complementarias (analítica de sangre con hemograma, bioquímica y coagulación) y si es posible, realización del índice tobillo/brazo (ITB).

- El tratamiento se realiza según la gravedad basada en clasificación IDSA (Infectious Diseases Society of America) adaptada por la Sociedad Española de Angiología y Cirugía Vascular (SEACV).

*PD leve: analgesia y descarga del pie si procede y remisión a consultas externas (CCEE) de Cirugía.

*PD diabético moderado: ingreso en el Servicio de Cirugía.

*PD grave: ingreso en el Servicio de Cirugía para amputación urgente y antibioticoterapia intravenosa.

\section{Fase 2: manejo en ingreso}

- Pruebas complementarias:

*Realización del ITB en el caso de que no se haya realizado en urgencias.

*Solicitud de analítica "perfil diabético" (hemograma, lípidos perfil, glucosa, ácido úrico, creatinina, GOT, GPT, gGT, HbA1c e índice microalbuminuria/creatinina). Si HbAlc elevada consultar a endocrino. Si insuficiencia renal consultar a nefrología.

*Ante sospecha de osteomielitis/pie de Charcot se solicitará una resonancia (RM) de pie.

- Tratamiento del PD moderado: si pulsos arteriales distales negativos, consultar con cirugía vascular del hospital de referencia. El antibiótico de elección es el ertapenem 1 gramo intravenoso cada $24 \mathrm{~h}$. Si hay gangrena seca con celulitis, tratamiento médico (antibiótico intravenoso). Si hay gangrena húmeda con/sin celulitis, amputación económica. Si existe absceso/esfacelos, drenar y desbridar tejido necrosado (tomar cultivo). Si hay úlcera neuropática con osteomielitis crónica, tratamiento médico (antibiótico intravenoso y descarga del pie). Si amputación mayor consultar a Rehabilitación.

- Cuidados del pie: pies en cama posición standard horizontal. Si celulitis y/o edema de pierna, valorar férula de Brown. En pie neuropático valorar zapato/bota de descarga para deambular (consultar a Ortopedia). En pie isquémico no elevar piernas en cama. Evitar povidona yodada en úlceras/heridas abiertas. 


\section{Fase 3: derivación del paciente susceptible de revascularización}

- Criterios de inclusión:

*Úlcera profunda isquémica/gangrena localizada (dedos, parte de pie, talón o planta) con/sin dolor en reposo.

* Pulsos arteriales distales (tibial posterior y pedio) negativos.

* No límite de edad, pero sí independiente para actividades básicas de la vida diaria (no encamado).

*ITB menor de 0,6 o mayor de 1,3.

*En caso de derivación de paciente, incluir: informe de alta/traslado con analítica, cultivo + antibiograma (si procede), vía periférica (o central si procede) y transferencia de imágenes ( $\mathrm{si}$ procede).

- Criterios de exclusión: PD leve, PD grave.

\section{Fase 4: selección del nivel de amputación en el} paciente no susceptible de revascularización

- Amputación de dedo o transmetatarsiana: paciente deambula, pulsos pedio o tibial posterior positivo.

- Amputación infracondílea: paciente deambula, pulso femoral positivo, temperatura de piel normal hasta tobillo.

- Amputación supracondílea: paciente no deambula, dolor isquémico en reposo, úlcera o gangrena, piel fría, lesiones cutáneas en pantorrilla.

Se denominó grupo AP a los pacientes recogidos desde el año 2010 a 2013, que corresponde al periodo anterior a la aplicación de un protocolo para el PD, y grupo DP a los pacientes registrados desde 2014 a 2018, periodo posterior a la aplicación del protocolo.

\section{Método estadístico}

Las variables cualitativas fueron expresadas mediante frecuencias y porcentajes, mientras que las variables cuantitativas con medias y desviaciones estándar. Se comprobó la asunción de distribución normal mediante el test de Shapiro-Wilk. Las comparaciones entre los datos se realizaron mediante la prueba t de Student para muestras independientes. Las variables cualitativas fueron analizadas usando la prueba de chi-cuadrado. Se consideró significancia estadística un valor de $p<0,05$. Los datos fueron analizados utilizando el programa IBMâ SPSSâ versión 22.0 para Mac (SPSSâ, Chicago, IL, USA).

\section{Resultados}

En el grupo AP se realizaron un total de 172 amputaciones por gangrena, úlcera o infección, sobre 115 pacientes, con una media de $43 \pm 2,7$, mientras que en el grupo DP se practicaron 111 amputaciones sobre 95 pacientes, media 22,2 \pm 9,9 amputaciones, con diferencias estadísticamente significativas (Figura 1). Desglosando por amputaciones mayores y menores, en el grupo AD se realizaron 57 amputaciones mayores (36\%) y 101 amputaciones menores (64\%); en el grupo DP 42 amputaciones mayores (38\%) y 69 amputaciones menores $(62 \%)$.

Según datos demográficos, la edad media del grupo AP fue de 78,3 $\pm 10,5$ años, la del grupo DP
Figura 1. Distribución del número de amputaciones realizadas cada año, antes (AP) y después del protocolo (DP).

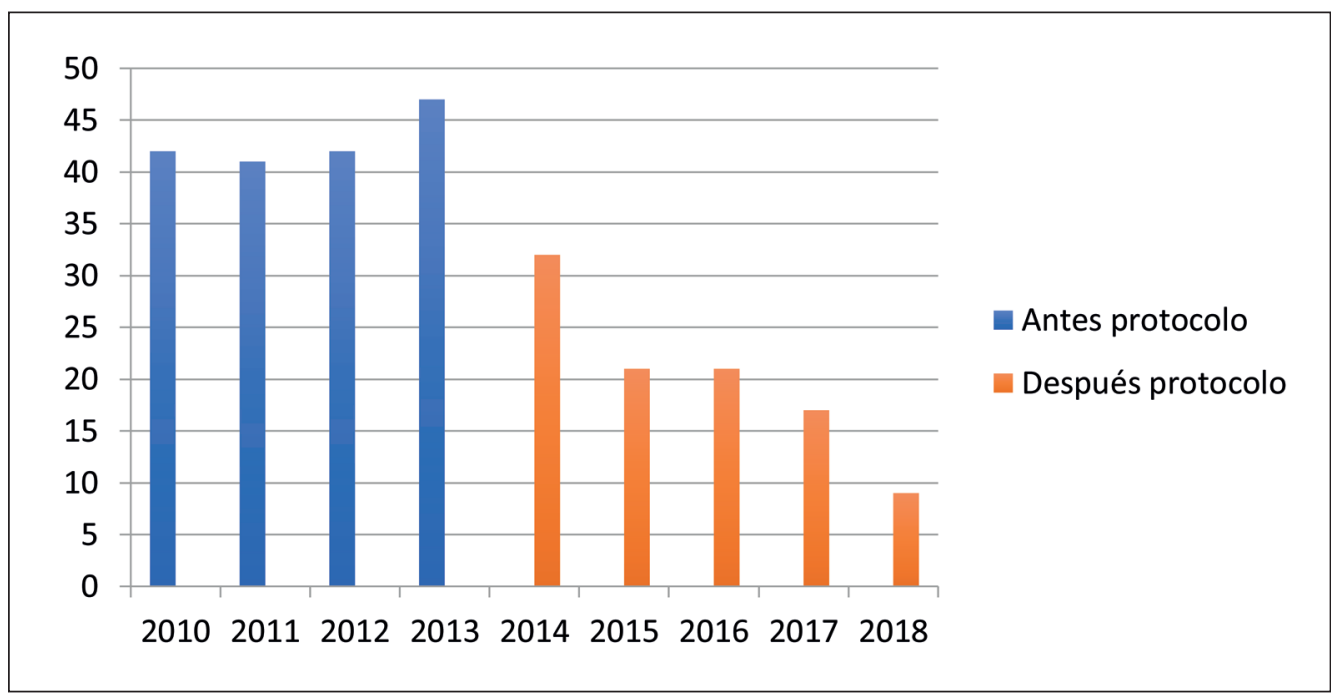

Rev. Cir. 2021;73(5):556-562 
$76,5 \pm 12,6$ años; en el grupo AP hay 64 hombres $(56 \%)$ y 51 mujeres $(44 \%)$, en el grupo DP 59 hombres $(62 \%)$ y 36 mujeres $(38 \%)$, sin observarse diferencias entre ambos grupos.

La estancia media en el grupo AP es de 8,8 días, la del grupo DP 8,7 días (NS). En el grupo AP, se han realizado en total 41 reamputaciones por mala evolución (35\%), en el grupo DP 15 reamputaciones, con diferencias estadísticamente significativas. En el primer mes se han reamputado 18 pacientes en el grupo AP, en el grupo DP 7, también significativo. En el grupo AP, los reingresos por mala evolución susceptibles de reamputación, suponen 10,5 días más de estancia hospitalaria, en el grupo DP es de 6,4 días (p 0,03).

Respecto a la solicitud de pruebas complementarias, en el grupo AP, se ha solicitado perfil diabético en 20 pacientes, en el grupo DP en $38(40,4 \%)$ con $\mathrm{p}<0,0001$. En el grupo AP, el valor medio de la $\mathrm{HbA1c}$ fue de 9,45 $\pm 1,9$ en el grupo DP 9,5 $\pm 2,1$ (rango normal 4,5\%-6,5\%). En el grupo AP la solicitud de angioTC/RM/doppler arterial de miembros inferiores se ha realizado en el grupo AP en 27 pacientes (17\%), en el grupo DP en 19 (NS).

En el grupo AP, durante el ingreso, se ha derivado al paciente al centro de referencia para valorar revascularización en 11 pacientes, en el grupo DP en 15 pacientes (NS) (Tabla 1).

En el momento de recogida de datos (noviembre de 2019 - enero de 2020) en el grupo AP, hubo 91 exitus $(57 \%)$, en el grupo DP 60 (63\%), con una esperanza de vida media tras la amputación (ya sea menor o mayor) de 19,5 meses. De los 51 pacientes en los que hemos podido averiguar por los datos de la historia clínica, la causa más frecuente de mortalidad, ha sido de origen cardiovascular.

\section{Discusión}

El pie diabético es una patología de manejo multidisciplinar donde, además de la atención primaria (AtP), participan muchas especialidades como el servicio de urgencias (SU), cirugía general, cirugía vascular, dermatología, traumatología, rehabilitación, podología, ortopedia, hospitalización a domicilio, enfermedades infecciosas, endocrinología, nefrología y oftalmología. Son diferentes los estudios internacionales que ya han demostrado que, atendiendo a estos pacientes en equipo, el número de amputaciones es mucho menor reduciéndose hasta en un 50-70 \% ${ }^{4}$. La atención multidisciplinar no significa que el paciente ingresa en un servicio y a continuación se realizan una serie de interconsultas
Tabla 1. Resultados de las variables estudiadas antes (AP) y después del protocolo (DP)

\begin{tabular}{|lccc|}
\hline \multicolumn{1}{|c}{ AP } & DP & p \\
Estancia (días) & $8,8 \pm 6,2$ & $8,6 \pm 5,6$ & NS \\
Reamputaciones & $41(35,7 \%)$ & $15(15,8 \%)$ & 0,001 \\
Reamputaciones 1 mes & $18(15,7 \%)$ & $7(7,4 \%)$ & 0,05 \\
Estancia prolongada (días) & $10 \pm 6,7$ & $6,4 \pm 4,5$ & 0,03 \\
Analítica perfil & $20(17,4 \%)$ & $38(40 \%)$ & 0,0001 \\
TC/RM/doppler arterial & $27(23,5 \%)$ & $19(20 \%)$ & $\mathrm{NS}$ \\
Derivación a centro de referencia vascular & $11(9,6 \%)$ & $15(15,8 \%)$ & $\mathrm{NS}$ \\
Éxitus & $91(79,1 \%)$ & $60(63,2 \%)$ & 0,01 \\
Total & 115 & 95 & \\
\hline
\end{tabular}

que la mayoría de las veces contestan profesionales sin interés por la enfermedad. Las unidades de pie diabético (UPD) deben estar adecuadamente formadas y actualizadas, con integrantes que comparten los mismos criterios, que se reúnen para discutir sobre las opciones terapéuticas de un caso concreto y que siguen las recomendaciones de los consensos internacionales y la literatura médica reciente ${ }^{5}$. En nuestro centro no existe UPD, sin embargo, hay gran comunicación con los servicios de infecciosos, dermatología, endocrino, rehabilitación, ortopedia y cirugía vascular de hospital de referencia.

El Departamento de Salud Valencia Arnau de Vilanova-Llíria abarca 316.981 habitantes, con una población diabética de 20.379 pacientes, todos ellos susceptibles de aparición de úlceras, infección o gangrena. En nuestro centro existe una unidad funcional de PD formado por dos cirujanos generales y una enfermera especializada en curas, que atienden y controlan a todos los pacientes con esta patología en las consultas externas (CCEE), procedentes de AtP, urgencias hospitalarias, poshospitalización, y también derivados de otras especialidades. Desgraciadamente, el mayor flujo de entrada de pacientes a nuestro hospital con PD es a través del SU, por iniciativa propia del mismo o remitido desde AtP por mala evolución, siendo el cirujano general el que asume íntegramente en ese momento esta patología (ya que no disponemos de cirujano vascular de guardia), el cual lo tratará, y decidirá alta con cita en CCEE, ingreso en nuestro servicio o derivación a centro de referencia para valorar revascularización. Por lo tanto, todos los cirujanos del departamento, 
aunque no pertenezcan a esa unidad, deben tener unos conocimientos básicos de PD, ya que a lo largo de la guardia es posible que tenga que enfrentarse a algún paciente con esta patología y tener que resolverla. Todo ello nos animó a realizar un protocolo de actuación cuya confección se desarrolló en el año 2013, implantándose en nuestro servicio de cirugía en enero de 2014.

Revisando la bibliografía española, hay muy pocas publicaciones originales sobre $\mathrm{PD}^{6-13}$. En el libro de la Asociación Española de Cirujanos $(\mathrm{AEC})^{14}$ se dedica apenas un capítulo; existe una guía de la $\mathrm{AEC}$ de cuidados del pie diabético ${ }^{15}$, pero no está actualizada. Por todo ello, nos estimuló a realizar este estudio y a intentar darle eco y más importancia a esta patología tan frecuente que no sólo supone el drama psicológico y físico por la amputación sino también un gran coste económico para la sanidad.

La primera fase del protocolo, atención en urgencias, está basada en el documento de consenso sobre las infecciones en el PD de $2012^{16}$. La segunda fase, atención en el ingreso, también se basa en el mismo, además en el manejo conservador de la osteomielitis ${ }^{17}$ y la guía práctica de la utilización de antisépticos en el cuidado de heridas ${ }^{18}$. La fase 3 , derivación a centro de referencia para valorar revascularización, se basa en el consenso de 2012 y en el consenso entre sociedades para el tratamiento de la arteriopatía diabética ${ }^{19} \mathrm{y}$, la fase 4 , selección nivel de amputación de miembro inferior por isquemia no susceptible de revascularización, está basada en la edición en español de 2011 del libro ACS Surgery ${ }^{20}$.

La tasa de amputaciones después de aplicar nuestro protocolo ha sido significativa, suponiendo un descenso de un $50 \%$ de media con respecto a antes de su existencia. Revisando la bibliografía, se demuestra que con la aplicación de un protocolo de actuación para el PD se reduce la tasa de amputación $^{21-22}$. La edad media del grupo AP fue de 78,3 años, la del grupo DP 77,3 años, mayor que otros estudios. En cuanto al sexo, en el grupo AP y PD predomina el varón $(56 \%-62 \%)$ con respecto a la mujer (44\%-38\%), similar a otros estudios ${ }^{21,23}$. La estancia media en el grupo AP ha sido de 8,8 días, la del grupo DP 8,7 días. En el estudio de De Alcalá $\mathrm{D}^{21}$ es algo mayor, 11 días. Monereo describe una estancia media de 19,8 días ${ }^{24}$. La tasa total de reamputaciones se ha reducido significativamente tras la aplicación del protocolo. En el grupo AP, se han realizado en total 41 reamputaciones por mala evolución $(35,7 \%)$, en el grupo DP 15 reamputaciones. En el primer mes se han reamputado 18 pacientes en el grupo AP, en el grupo DP 7, también significativo, probablemente por haber elegido mejor el nivel de amputación con la aplicación del protocolo. En el grupo AP, los reingresos por mala evolución susceptibles de reamputación, suponen 10,5 días más de estancia hospitalaria, en el grupo DP es de 6,4 días, significativa.

En el grupo DP ha aumentado de forma significativa la solicitud de perfil diabético (de un $12 \%$ a un $40,4 \%$ ). Hay que destacar que en el $71 \%$ del total, tenían la HbA1c alterada, con una media de 9,5\% (rango normal 4,5\%-6,5\%). En nuestro centro, si la HbA1c estaba alterada se consulta con endocrino en el mismo ingreso. Se ha visto que con un control glucémico estricto mejoran las lesiones del PD y se reduce la tasa de amputaciones en un $35 \%{ }^{25-27}$. No ha habido diferencias en cuanto a pacientes derivados al centro de referencia para valorar revascularización, y tampoco en la solicitud de pruebas radiológicas complementarias específicas antes y después del protocolo. Recientemente, hemos creído conveniente añadir en el protocolo la solicitud de TC/RM al paciente que ingresa por $\mathrm{PD}$, ya que la mayoría de los pacientes suelen tener un componente vascular añadido además del neuropático y/o infeccioso. Un estudio concluyó que, tras la aplicación de la vía clínica, aumentó de forma significativa el número de pacientes que recibieron examen arteriográfico ${ }^{21}$. La mortalidad ha sido similar en los dos grupos, con una esperanza de vida media tras la amputación (ya sea menor o mayor) de 19,5 meses, siendo la más frecuente la cardiovascular. Un estudio relata que la mortalidad a cinco años después de la amputación relacionada con la diabetes es de hasta un $60 \%$, que es más alta que para ciertas neoplasias malignas ${ }^{28}$. La causa más frecuente de mortalidad en la bibliografía consultada también es la cardiovascular ${ }^{29}$. Finalmente, y como hemos comentado al principio de la discusión, volvemos a destacar que es fundamental que la atención al paciente con PD sea llevada por un equipo multidisciplinar para la buena evolución del mismo ${ }^{1,4,5,27,28,30}$.

\section{Conclusiones}

El PD es una patología de manejo multidisciplinar, donde el cirujano general constituye un pilar fundamental, sobre todo en los centros donde no se dispone de cirujano vascular. La existencia de un protocolo para PD es fundamental, ya que reduce claramente la tasa de amputaciones y reamputaciones. Además, es necesario un contacto más directo con AtP en el manejo del PD. 


\section{Responsabilidades éticas}

Protección de personas y animales. Los autores declaran que para esta investigación no se han realizado experimentos en seres humanos ni en animales.
Confidencialidad de los datos. Los autores declaran que en este artículo no aparecen datos de pacientes.

Conflictos de interés: no hay.

\section{Bibliografía}

1. Pérez A. Abordaje multidisciplinar. Organización de una unidad de pie diabético. Mon Act Soc Esp Med Cir Pie Tobillo 2018;10:3-9.

2. Prompers L, Huijberts M, Apelqvist J, Jude E, Plaggesi A, Bakker K, et al. High prevalence of ischaemia, infection and serious comorbidity in patients with diabetic foot disease in Europe. Baseline results from the Eurodiale study. Diabetología 2007;50:18-25.

3. Lavery LA, Armstrong DG, Wunderlich RP, Tredwell J, Boulton AJ. Diabetic foot síndrome: Evaluating the prevalence and incidence of foot pathology in Mexican American san non-Hispanic whirtes from a diabetes disease management cohort. Diabetes Care 2003;26:1435-8.

4. Sumpio BE, Armstrong DG, Lavery LA, Andros G. The role o fan interdisciplinary team approach in the management of the diabetic foot. J Vasc Surg. 2010;51:15046.

5. Aragón J. ¿Son útiles las unidades de pie diabético? Med Clin. (Barc) 2014;142:208-10

6. De Alcalá D, Aguayo JL, Flores B, Morales G, Pérez-Abad JM, Alarte JM. Resultados de la hospitalización en pacientes con pie diabético. Cir Esp. 2003;74:92-6

7. De Alcalá D. Tratamiento de la infección en el pie diabético. Cir Esp. 2004;76:9-15.

8. Lázaro JL, García E, Beneit JV, Martínez FR. Estudio aleatorizado y comparativo de un apósito de colágeno y celulosa oxidada regenerada en el tratamiento de úlceras neuropáticas de pie diabético. Cir Esp. 2007;82:27-31.

9. Sepúlveda G, Espíndola M, Maureira M, Sepúlveda E, Fernández JI, Oliva C, et al. Curación asistida por presión negativa comparada con curación convencional en el tratamiento del pie diabético amputado.
Ensayo clínico aleatorio. Cir Esp. 2009;86:171-7.

10. Dzieciuchowicz L, Espinosa G, Grochowicz L. El sistema de cierre asistido al vacío en el tratamiento del pie diabético avanzado. Cir Esp. 2009;86:2138

11. Sellés R, Rueda C, Primo V, Martínez A, Asencio F. Papel del cirujano general en el diagnóstico y tratamiento precoz del pie de Charcot. Cir Esp. 2015;93:320-5.

12. Caimari F, González C, Ramos A, Chico A, Cubero JM, Pérez A. Eficacia de un programa de tratamiento de la hiperglucemia en un Servicio de Cirugía Vascular supervisado por Endocrinología. Cir Esp. 2016;94:392-8.

13. Marcos L, Mateos E, Velescu A, Díaz C, Lacambra M, Clará A. Historia natural del paciente sometido a una amputación infracondílea: análisis de la supervivencia y protetización durante 2 décadas. Disponible en https://doi.org/10.1016/j. ciresp.2019.11.007.

14. De Alcalá D. Pie diabético. En: Parrilla Paricio P, Landa García JI. Cirugía AEC $2^{\mathrm{a}}$ edición. Asociación Española de Cirujanos. Madrid: Panamericana S. A., 2010;103:1085-93.

15. De Alcalá D. Cuidados del pie diabético. Un enfoque multidisciplinario. Asociación Española de Cirujanos. Madrid: Arán S.A. 2001.

16. Blanes JL, Clará A, Lozano F, Alcalá D, Doiz E, Merino R, et al. Documento de consenso sobre el tratamiento de las infecciones del pie diabético. Angiología 2012;64:31-59.

17. Osteomielitis pie diabético ¿es posible manejo conservador? Jordano-Montáñez Q, Muñiz-Tatay M, Viadé-Julià J, JaenManzanera A, Royo-Serrando J, CuchíBurgos E, et al J. Enferm Infecc Microbiol Clin. 2014;32:555-9.

18. Guía práctica de la utilización de antisépticos en el cuidado de heridas. Disponible en http://www.salvatbiotech.
com/Content/Media/45fcbaa337de402fbc1 152e0947ccce5/GuiaAntisepticos.pdf

19. Consenso entre sociedades para el tratamiento de la arteriopatía periférica. Journal of Vascular Surgery 2007;45S:5171. Disponible en http://www.ulceras.net/ publicaciones/TASCII.pdf

20. Pevec WC. Amputación de extremidades inferiores por isquemia. ACS Surgery Principles \& Practice. Focus on Orthopedics. Edición 2011 en español.

21. De Alcalá D, Aguayo JL, Morales G, Aguirán LM, Illán F. Impacto de una vía clínica para el pie diabético en un hospital general. An Med Interna 2004;21:420-24.

22. Crane M, Werber B. Critical pathway approach to diabetic pedal infections in a mulidisciplinary setting. J Foot Ankle Surg. 1999;38:30-3.

23. Marcos L, Mateos E, Velescu A, Díaz C, Lacambra M, Clará A. Historia natural del paciente sometido a una amputación infracondílea: análisis de la supervivencia y protetización durante 2 décadas. Disponible en https://doi.org/10.1016/j. ciresp.2019.11.007

24. Monereo S, Pavón I, Vega B, Elviro R, Durán M. Complicaciones de la diabetes mellitus: impacto sobre los costes hospitalarios. Endocrinología 1999;46:559.

25. Caimari F, González C, Ramos A, Chico A, Cubero JM, Pérez A. Eficacia de un programa de tratamiento de la hiperglucemia en un Servicio de Cirugía Vascular supervisado por Endocrinología. Cir Esp. 2016;94:392-8.

26. Dissanayake A, Vandal AC, Boyle V, Park D, Milne B, Grech R, et al. Does intesnsive glycaemic control promote healing in diabetic foot ulcers? - a feasibility study. BMJ Open 2020; 10:e029009. DOI: 10.1136/ bmjopen-2019-029009.

27. Everett E, Mathioudakis N. Update on management of diabetic foot ulcers. Ann NY Acad Sci. 2018;1411:153-65. 


\section{ARTÍ́CULO ORIGINAL}

28. Mavrogenis AF, Megaloikonomos $\mathrm{PD}$, Antoniadou T, Igoumenou VG, Panagopoulos GN, Dimopoulos L, et al. Current concepts for the evaluation and management of diabetic foot ulcers. EFORT Open Rev. 2018;3:513-25.
29. Rubio JA, Jiménez S, Álvarez J. Características clínicas y mortalidad de los pacientes atendidos en una Unidad Multidisciplinar de Pie Diabético. Endocrinol Diabetes Nutr. 2017; 64:241-9.
30. Brocco E, Ninkovic S, Marin M, Whisstock C, Bruseghin M, Boschetti $\mathrm{G}$, et al. Diabetic foot management: mutidisciplinary approach for advanced lesión rescue. J Cardiovasc Surg. 2018;59:670-84. 